\title{
Analysis of Visual Language for Stop-motion Animations
}

\author{
Cang Minnan ${ }^{1}$; Tan Yizhe $^{2} ;$ Xie Shenglu \\ ${ }^{1} \mathrm{Xi}$ 'an university of technology, yanxiang road, yanta district, $\mathrm{xi}$ 'an city, shaanxi province, $\mathrm{CHN}$ \\ ${ }^{2} \mathrm{Xi}$ 'an university of technology, yanxiang road, yanta district, $\mathrm{xi}$ 'an city, shaanxi province, $\mathrm{CHN}$ \\ ${ }^{3} \mathrm{Xi}$ 'an university of technology, yanxiang road, yanta district, $\mathrm{xi}$ 'an city, shaanxi province, $\mathrm{CHN}$
}

\begin{abstract}
This paper explores the visual languages for stop-motion animation from the perspective of artistic expression and different processes of stop-motion animation production. In terms of the application of role performance, modeling effect and true feelings brought by materials, the paper analyzes the language rules and relations among these three aspects and shows the laws by combining some artistic theories and animation techniques.
\end{abstract}

\section{Introduction}

Clear, vivid and abundant visual language, which is medium of expressing various regional cultures and emotions, is at the center of discussion and experiments among animators and practitioners involved in animation.

One important feature of visual language is reflecting visual culture through static and dynamic images, thus enabling individuals from various groups and regions to accept and understand behaviors, acts and backgrounds of characters as well as events and plot in stories.

The above-mentioned feature is rather complicated and changeable owing to the specialty in making and filming of frame-by-frame animation, which can be roughly divided into technical filming and lens language according to processing and visual language of stop-motion animation. Lens language is composed of body emotions and lens.

To analyze stop-motion animation, the key lies in role performance. This paper is directed at studying modeling effects in different stages and exploring the function of visual language in shaping characters.

In making and filming stop-motion animation, a visual language system in line with the standard of stop-motion animation can be concluded through several steps. Firstly, we should analyze the relation and distinction between stop-motion animation and other forms of animation, including two-dimensional animation and three-dimensional animation. Secondly, we should study stop-motion animation making to analyze specialty of performance, modeling and photographing in each stage of stop-motion animation. Thirdly, we should blend body and behavioral languages, realistic depiction, application of materials and artistic modeling aesthetics together. These steps can also be applied to teaching and practicing, improving students' analytic ability of camera language and induction ability, and frosting students' creativity.

\section{Application of Role Performance}

Stop-motion visual language is to vitalize puppets, which are molded by clay, silicone, foaming rubber and resin through a series of complicated processes, using the filming technique of frame by frame. The key to reflecting visual language in films is animation modeling and law of motion.

Apart from following script and character setting, modeling characteristics of roles should be in line with performance requirements in animation. This is because that camera language is firstly expressed by performance of roles. In filming, a conflict between intention of animator and technique limit always exists.

Through a large number of practices, role performance is reflected and applied in different degrees in animation making. For instance, when molding the role, according to filming functions, a combination of assembly method and casting method can effectively simplify filming based on shooting scripts. (figure 1) 

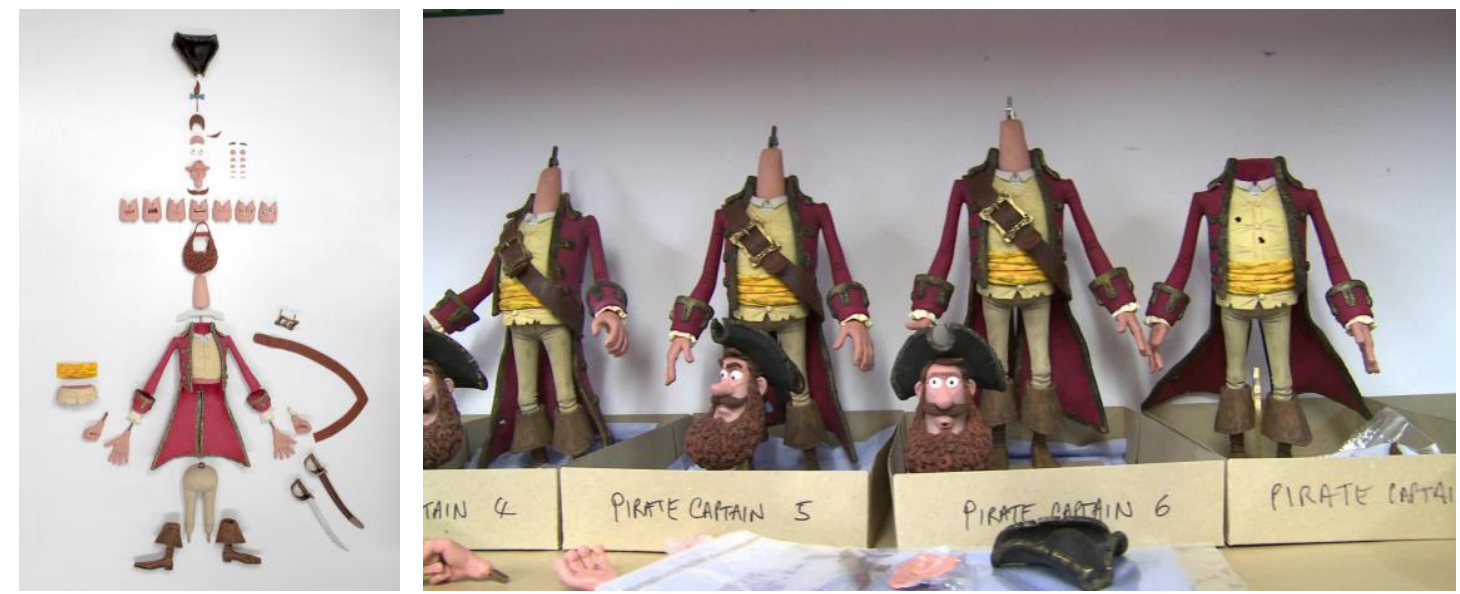

(figure 1): In The Pirates! Band of Misfits produced by Aardman Animation, using casting method, the body of the role Captain was molded by silicone, and the head and mouth by clay and foaming rubber, highlighting modeling details, as well as protecting puppets from possible surface deformation and fingerprints in posing.

\section{Role Modeling Effect}

Whether in celluloid sheet, computer animation or any stop-motion animation, role modeling exists and affects final presentation of film.

Requirements for role modeling in stop-motion animation are quite distinct from that for two-dimensional and three-dimensional animation. Firstly, roles are molded by actual materials. Secondly, atmosphere of film is created through puppet performance, showing an both static and dynamic art. Thirdly, visual effects of the plot of animation are achieved by using 3 methods and 5 techniques in montage with stop-motion two-dimensional pictures.

The contents and demonstration of effects feature three aspects:

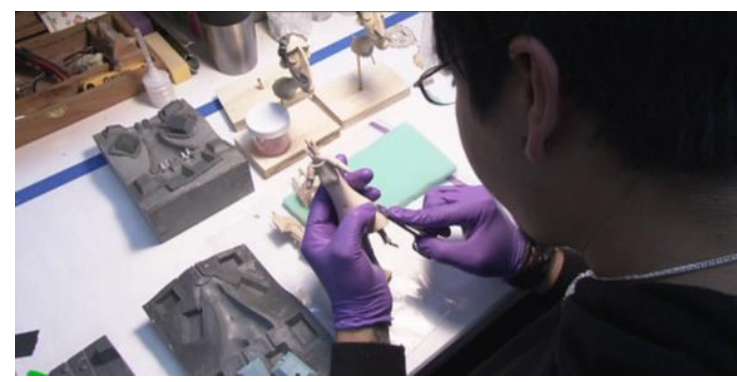

\subsection{Static form. Standards:}

\subsubsection{Roles created must combine accurateness with abstraction;}

\subsubsection{The personality of roles based on prototype should be beyond limits of external shape, seeking recognition from all cultures;}

\subsubsection{The material chosen should be in accordance} with filming requirements. Skeleton of roles, foaming rubber, silicone and clay should be able to stand banding back and forth without damage or deformation; (Figure 2)

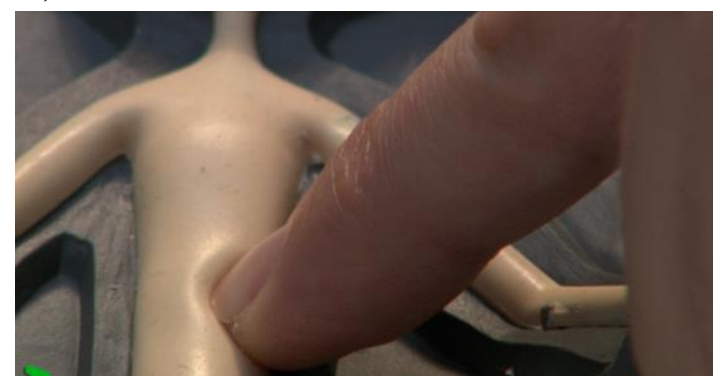

(Figure 2): In Coraline and The Secret Door(2009) directed by Henry Selick, all roles were molded by silicone, which maintained its own toughness and met the need of frame by frame filming of stop-motion animation. Joints of roles would not damage or split when bending back and forth. At the same time, using casting method, period of making puppets was shortened. More importantly, uniformity of role modelling could be ensured in duplication of the same puppets. 


\subsubsection{To facilitate filming, performance of roles' facial}

expression should be put into consideration in making head skeleton of puppets. (Figure 3 and 4)
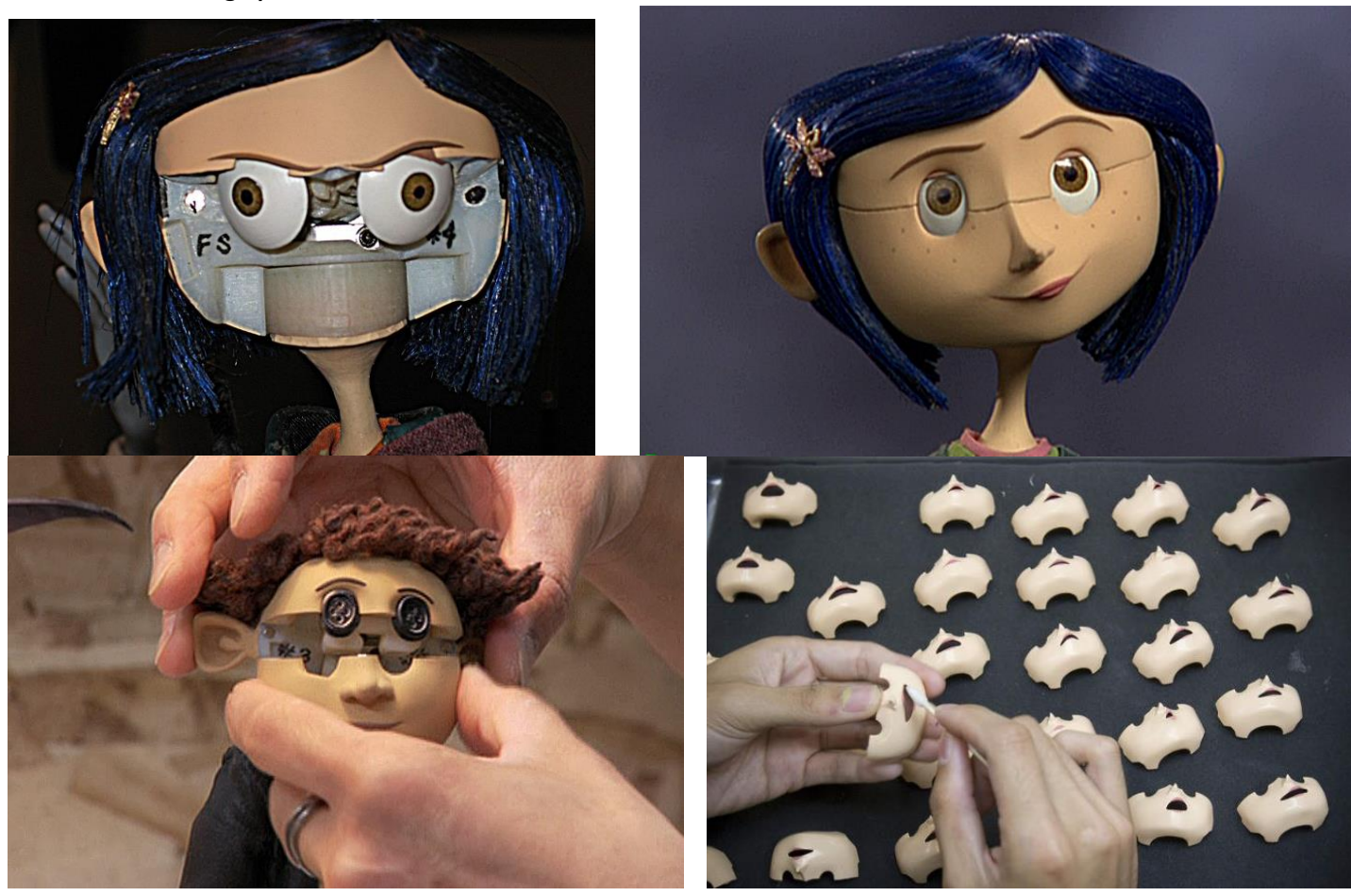

(Figure 3): As animation takes off, clay figures in Wallace and Gromit can hardly satisfy people's visual demand for roles. Besides delicacy and exquisiteness of roles, higher standards are seen in traditional animation making like stop-motion animation. As producer of both Kubo and The Two Strings and Coraline and The Secret Door, American Laika Entertainment, set up in 1990, has been committed to making and filming of stop-motion animation. In movies released respectively in 2009 and 2017, to film mouth movements, resin was used with the help of 3D printing. With regard to synchronization of sound and picture, thousands of faces with various facial expressions were prepared to achieve dialogues between characters. The new method was based on prior $3 \mathrm{dsmax}$ Modeling and 3D printing, requiring more complicated interior skeleton of puppets.
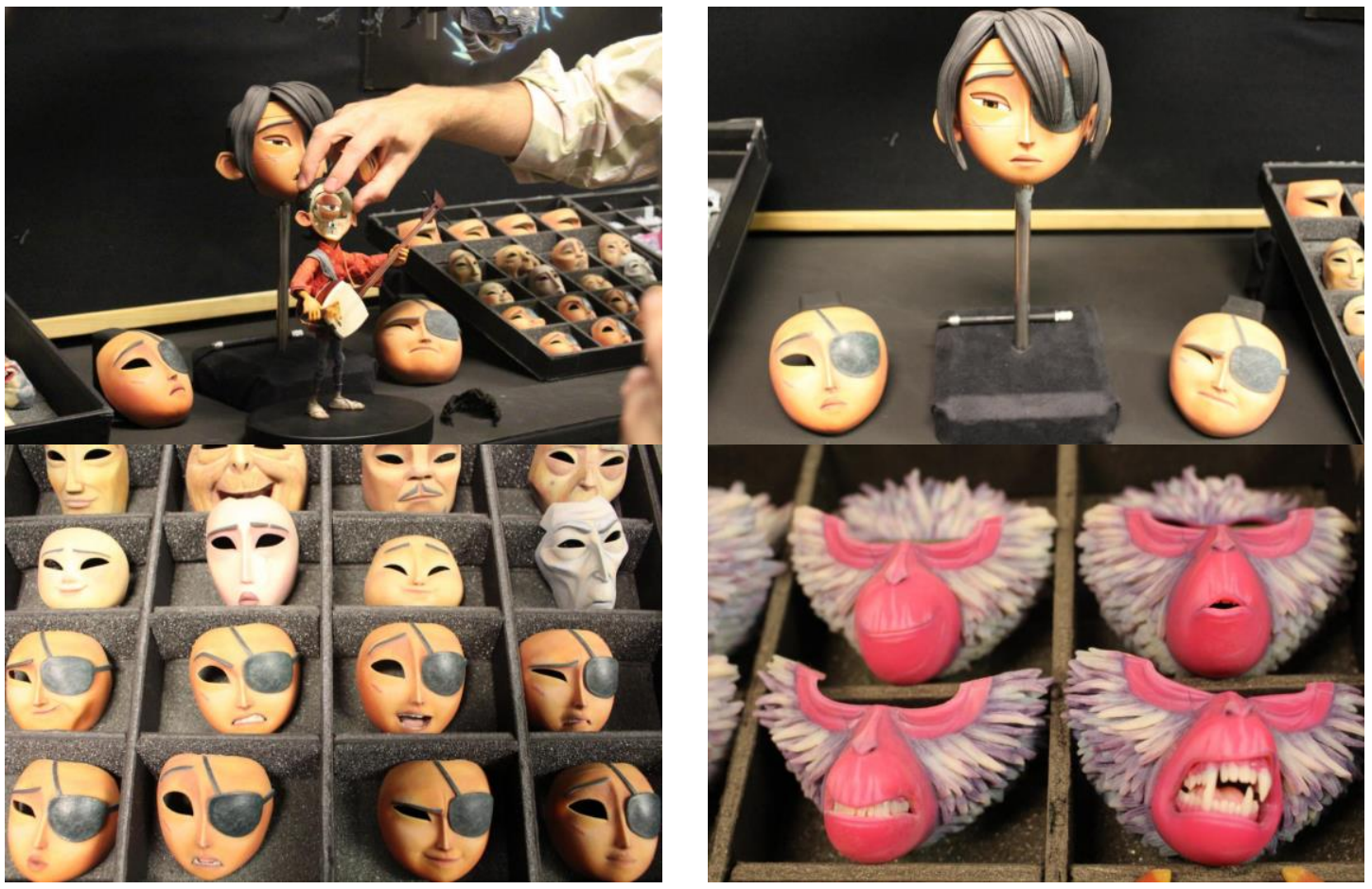

(Figure 4): With more than 70 scenes, as long as 5 years were spent to make Kubo and The Two Strings. On average, 2-5 seconds were shot every day. Before Kubo, who himself had a combination of 48 million facial expressions, Laika Entertainment's highest record was 1.5 million. Though filming method being stop-motion, Kubo and The Two Strings used three-dimensional technology and it combines higher shooting frame rate with more complex roles and scenes as well as more exquisite final processing. Judging 
from pictures, people can hardly believe that it is a stop-motion animation shot frame by frame. At the same time, this film provides all practitioners with a new idea about the future of stop-motion animation in a digital world.

\section{puppets;}

\subsection{Dynamic form. Standards:}

\subsubsection{The body structure of puppets should comply with moving characteristics of roles and law of motion;}

\subsubsection{Performance ability of roles is a must;}

\subsubsection{Molding craftsmen and animators should have basic ability and cultural cultivation to manipulate}

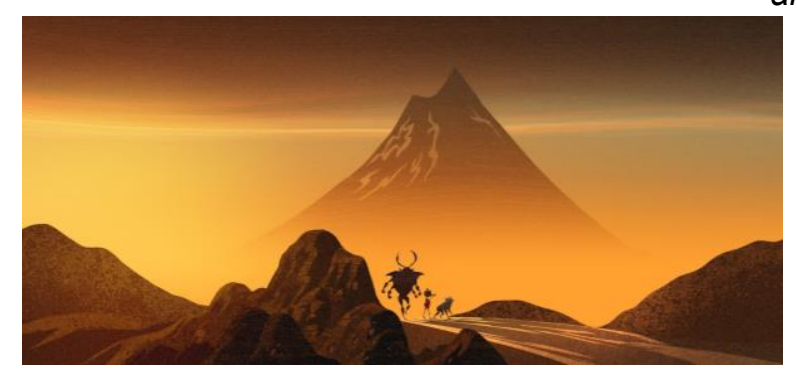

\subsection{Photographing (role modeling and performance effect of stop-motion pictures). Standards:}

3.3.1 The sets should reflect the relationship between roles created and scene and story;

3.3.2 Light arrangement not only satisfies the need of shaping characters externally and internally, but also creates atmosphere for performance; (Figure 5)

(Figure 5): screenshot of scene setting in Kubo and The Two Strings

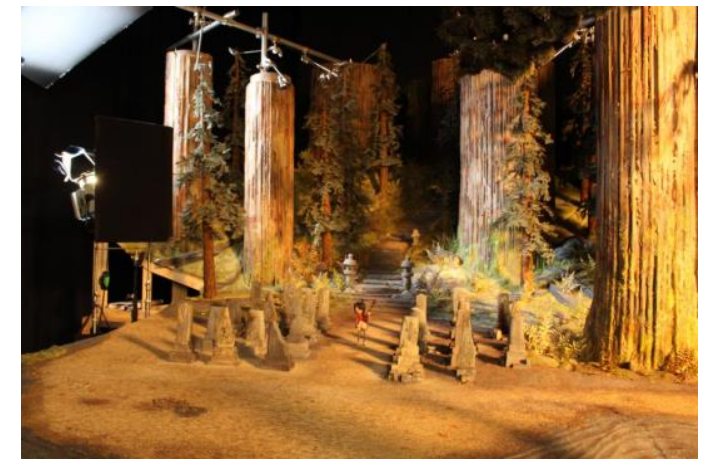

Kubo and forests in Kubo and The Two Strings

3.3.3 Role performance should follow ups and downs of the story, creating atmospheres and expressing emotions according to different visual and psychological effects the director wishes audience to feel;

\subsubsection{The application of technique of montage,} including juxtaposition, parallelism, contrast, symbolism and repetition, should comply with people's habit of association and memory. Visual characters in films should be shaped accurately through the use of recall, expectation, interposition, imagination and flashback.

\section{True Feelings Brought about by the Materials}

Materials used for characters and props will produce a desired effect in stop-motion animation. Feelings brought

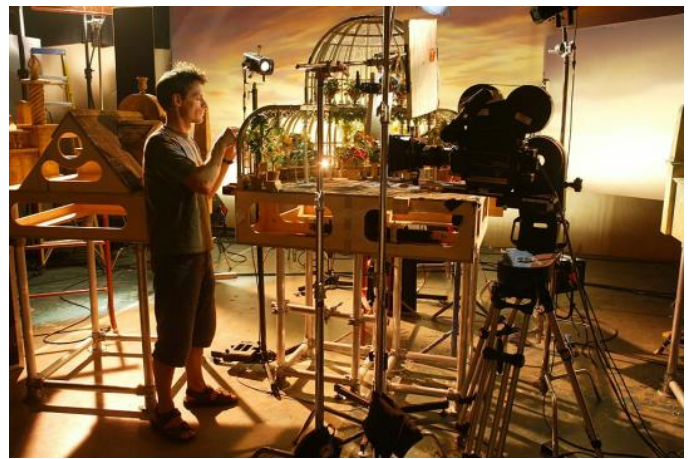

Light arrangement at dusk in Wallace \& Gromit in The Curse of the Were-Rabbit

by materials stem from its realistic feature and shaping beauty.

First of all, authenticity of materials characteristic to stop-motion animation is unparalleled compared to two-dimensional and three-dimensional animation. As a result, application of materials produces realistic beauty.

Aesthetics brought by materials applied (materials used in role modeling) creates a sense of beauty visually. Besides embodying texture of roles, visual effects brought by materials put a soul into roles, giving a life to roles who now own feelings, minds, emotions, willingness and behaviors. The research of Digital Performance of Shadow Play Using Frame by Frame Animation Technique is committed to broaden range of performance carriers for shadow play. Combining shadow play with frame by frame photographing technique in stop-motion animation not only enlarges exhibition space for shadow play, but also raises a new idea for protection of intangible cultural heritage like shadow play. (Figure 6) 


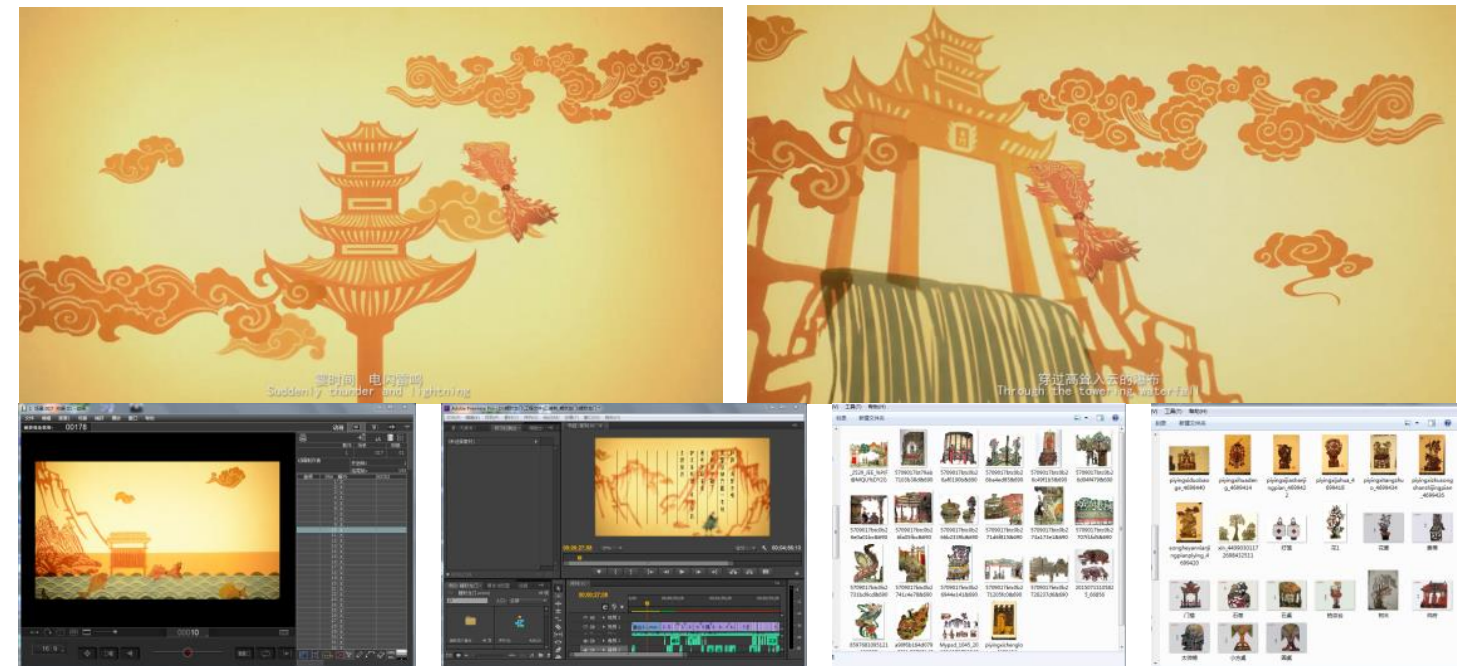

(Figure 6): Using frame by frame animation technique, Big Splash, a folktale about a carp leaping over Longmen, is an experimental film based on folk shadow play art, with setting and materials being in accordance with that in shadow play. In a digital world, a new way to protect and inherit traditional cultures and intangible cultural heritage can be achieved if combined with digital forms.

Artistic shaping beauty distinguishes stop-motion animation from hand painting and computer animation. Because of characteristics of materials, sometimes stop-motion animation is endowed with an austere but not rigid beauty. Like exquisite skeleton and skin made by silicone, shaping and application of materials based on new techniques produce brand new Gothic aesthetics. An irresistible charm beyond bodies, emotions, behaviors and shots is one of artistic language features of stop-motion animation, keeping it immortal and expressing thoughts of animators.

\section{Conclusion}

As media diversifies, the visual language of stop-motion animation combined with digital technology is more vivid and abundant. Roles come to life only when ideology and artistry of works are well balanced according to different functions of stop-motion animation.

The innovation in cultural system and institution, which is difficult, is the key to the innovation of visual language. Animators and practitioners should put emphasis on both market breakthrough and technological support, combing with new technique and new method. Continuous innovation is indispensable if we strive to enrich visual effects of stop-motion animation.

"We choose the most laborious form of animation which has beauty and warmness other forms of animation are not endowed with. We strive because we love stop-motion animation," said Stravis Knight, CEO of Laika Entertainment, also a director.

\section{Acknowledgments}

The annual art project of Shaanxi province granted by the provincial department of culture of Shaanxi: Digital Performance of Shadow Play Using Frame by Frame Animation Technique

\section{References}

1. Chen Mai, Stop Motion Animation Techniques [ M]. China Renmin University Press, 2005.8.

2. Basic Course of British Animation Design [M]. Shanghai People's Fine Arts Publishing House, 2005.6.

3. Wang Jiamin, Introduction to Chinese Art Design [M]. China Federation of Literary and Art Circles Publishing Corporation, 2006.10

4. Zhu Hui, Audio-Visual Language and Animation Footage [M]. Tsinghua University Press, 2009.11

5. David Cohen, Visual Language of British Series of Basics of Design $[\mathrm{M}]$. Shanghai People's Fine Arts Publishing House, 2012.1

6. Wang Zhimin, Zhao Bin, Cinematic Linguistics $[\mathrm{M}]$. Peking University Press, 2015.1 